\title{
BLOW-UP IN A SYSTEM OF PARTIAL DIFFERENTIAL EQUATIONS WITH CONSERVED FIRST INTEGRAL. PART II: PROBLEMS WITH CONVECTION*
}

\author{
C. J. BUDD ${ }^{\dagger}$, J. W. DOLD ${ }^{\dagger}$, AND A. M. STUART ${ }^{\ddagger}$
}

\begin{abstract}
A reaction-diffusion-convection equation with a nonlocal term is studied; the nonlocal operator acts to conserve the spatial integral of the unknown function as time evolves. The equations are parameterised by $\mu$, and for $\mu=1$ the equation arises as a similarity solution of the Navier-Stokes equations and the nonlocal term plays the role of pressure. For $\mu=0$, the equation is a nonlocal reaction-diffusion problem. The aim of the paper is to determine for which values of the parameter $\mu$ blow-up occurs and to study its form. In particular, interest is focused on the three cases $\mu<\frac{1}{2}$, $\mu>\frac{1}{2}$, and $\mu \rightarrow 1$.

It is observed that, for any $0 \leq \mu \leq \frac{1}{2}$, nonuniform global blow-up occurs; if $\frac{1}{2}<\mu<1$, then the blow-up is global and uniform, while for $\mu=1$ (the Navier-Stokes equations) there are exact solutions with initial data of arbitrarily large $L_{\infty}, L_{2}$, and $H^{1}$ norms that decay to zero. Furthermore, one of these exact solutions is proved to be nonlinearly stable in $L_{2}$ for arbitrarily large supremum norm. An understanding of this transition from blow-up behaviour to decay behaviour is achieved by a combination of analysis, asymptotics, and numerical techniques.
\end{abstract}

Key words. blow-up, nonlocal source term, conserved integral, Navier-Stokes equations, exact solutions, nonlinear stability

AMS subject classifications. 35B30, 35B35, 35K57, 65N50

1. Introduction. In this paper, we continue the study initiated in [BDS1] of the blow-up of solutions of parabolic partial differential equations (PDEs) in which the spatial integral of the unknown function is constrained to be zero. In particular, we incorporate the effect of convection to obtain a system of PDEs that arises as a perturbation of a similarity solution of the Navier-Stokes equations in an infinitely long channel. We find that, while there is no evidence that the solutions of the Navier-Stokes equations considered here blow up (and indeed all the evidence indicates otherwise), the slightly perturbed equations all exhibit blow-up. One of the major issues we consider is how this change in behaviour arises. It appears that the amount of convection is critical in determining if blow-up occurs; this justifies our study of a parameterised system of equations linking the nonlocal reaction-diffusion (no convection) equation to the Navier-Stokes equations. We believe that it is interesting to understand the sense in which the solutions of Navier-Stokes equations are affected by small perturbations to the governing equations and, for this reason, we study the parameterised family, including the Navier-Stokes equations.

To do this, consider the following initial-boundary value problem: Find $u(x, t)$, $v(x, t)$, and $K(t)$ satisfying

$$
\begin{gathered}
u_{t}+\mu v u_{x}=u_{x x}+u^{2}-K^{2}, \quad 0<x<1, \quad 0<t<T \\
v_{x}=u, \quad 0<x<1, \quad 0<t<T
\end{gathered}
$$

together with boundary conditions, for $0<t<T$,

$$
u_{x}(0, t)=u_{x}(1, t)=v(0, t)=v(1, t)=0
$$

* Received by the editors June 9, 1992; accepted for publication (in revised form) July 12, 1993.

$\dagger$ School of Mathematics, University of Bristol, Bristol BS8 1TW, United Kingdom.

¥ School of Mathematical Sciences, University of Bath, Bath BA2 7AY, United Kingdom. Current address, Program in Scientific Computing and Computational Mathematics, Division of Applied Mechanics, Durand Building, Stanford University, Stanford, California 94305-4040. 
and initial condition

$$
u(x, 0)=u_{0}(x)
$$

Here $T$ is the blow-up time. This system is formally third order in the spatial variables, but has four boundary conditions that are compensated for by the fact that $K(t)$ is unknown. Integrating (1.2) and applying the boundary conditions on $v(x, t)$ shows that

$$
I:=\int_{0}^{1} u(x, t) d x=0, \quad 0<t<T,
$$

and then integration of (1.1) using parts shows that

$$
K^{2}=(1+\mu) \int_{0}^{1} u^{2}(x, t) d x
$$

Thus the initial data is chosen to be compatible with (1.5). When $\mu=1$, the above system is equivalent to a similarity solution of the Navier-Stokes equations and $K(t)$ is closely related to pressure.

In [BDS1] we considered the case where $\mu=0$. Problem (1.1) is then a reactiondiffusion equation with a conserved first integral corresponding to a conservation of the total mass of the system. Such systems arise naturally in models of chemotaxis in mathematical biology [Ch] and also arise in studies of phase separation in binary alloys, particularly, the nonlocal Allen-Cahn equations described in [RS]. In [BDS1] we show that if the initial data has a maximum at the origin, is monotone decreasing in $x$, and large enough, then blow-up occurs so that $u(0, t)$ becomes infinite in a finite time. Moreover, this blow-up is global in the sense that $|u(x, t)| \rightarrow \infty$ as $t \rightarrow T$ for all $x \in[0,1]$. However, the blow-up is also nonuniform in the sense that $u(x, t)$ blows up most rapidly at the origin. Smaller initial data lead to solutions that decay to zero as $t \rightarrow \infty$. Similar thresholds on the initial data leading to blow-up have been observed for the chemotaxis equations. In this paper, we extend these results to study the evolution of problem (1.1) in the case where $0<\mu \leq 1$. The particular case of $\mu=1$ arises as a similarity reduction of the Navier-Stokes equations, and details of this relation are given in $\S 2$. Setting $\mu<1$ in (1.1) varies the amount of convection in the problem and thus gives an insight into the relationships between the dynamics of a class of solutions of the Navier-Stokes equations and the dynamics of a reaction-diffusion problem. Other studies of blow-up in the presence of convection for rather different systems not directly related to the Navier-Stokes equations are given in $[\mathrm{ABG}]$ and $[\mathrm{LAF}]$.

Our interest in this problem for $\mu$ close to 1 follows from the (numerical) observation that, when $\mu<1$, certain classes of initial data lead to finite time blow-up, whereas, when $\mu=1$, there is no numerical evidence of blow-up; furthermore, there are exact solutions with initial data of arbitrarily large $L^{\infty}, L^{2}$, or $H^{1}$ norms on $(0,1)$ that decay to the stable equilibrium $u \equiv 0$ as $t \rightarrow \infty$. One of these solutions is proved in $\S 5$ to be stable for arbitrary supremum norm. Thus, while the Navier-Stokes equations together with the geometry and boundary conditions considered in $\S 2$ appear to yield bounded solutions, this bounded behaviour may disappear under arbitrarily small perturbations of the original equations.

For more general values of $\mu<1$, problem (1.1) becomes interesting because the precise structure of the blow-up set and the nature of $u(x)$ close to blow-up depends 
subtly upon the value of $\mu$. If $\mu \leq \frac{1}{2}$, then the blow-up is nonuniform in the sense of the above definition. However, if $\frac{1}{2}<\mu<1$, then the blow-up is uniform in the sense that as $t \rightarrow T$, then $u\left(x_{1}, t\right) / u\left(x_{2}, t\right)=\mathcal{O}(1)$ for general $x_{1}, x_{2} \in[0,1]$. A crude physical motivation of this behaviour for a combusting system is as follows: If the convection is weak, then a hot spot forms at a unique point. For stronger convection, the heat from the hot spot is convected to the whole of the material, causing global blow-up. However, if the convection is too strong, then the hot spot can no longer generate enough heat and the system does not blow up. Thus problem (1.1) acts as a simple model for a variety of systems where a transition from local to uniform blow-up and then to decay occurs. To understand this transition and, in particular, the limit of the behaviour as $\mu \rightarrow 1$, we establish the following formal and rigorous propositions by a combination of asymptotics, computations, and analysis.

Formal Proposition I (steady state). Let $\mu<1$ and $|\mu-1| \ll 1$. Then, for any integer $n$, problem (1.1)-(1.4) has unstable steady-state solutions $u_{s}(x)$, with $n$ zeros such that

$$
u_{s}(x) \approx \frac{\left(n^{2} \pi^{2}\right)}{2(1-\mu)} \cos (n \pi x)+\mathcal{O}(1), \quad K \approx \frac{n^{2} \pi^{2}}{2(1-\mu)} .
$$

If $n=1$, the solution has a boundary layer of "width" $\mathcal{O}(1-\mu)^{1 / 2}$ at $x=1$. This boundary layer is such that

$$
u_{s}^{\prime \prime}(1) \approx-\frac{\pi^{4}}{2(1-\mu)}+\pi^{4} \log (1-\mu)+\mathcal{O}(1)
$$

For more general $n$, the resulting solution $u_{s}(x)$ is a reflection and a rescaling of the corresponding solution when $n=1$, and there is a sequence of boundary layers at the points $x=(2 k+1) / n$.

Although the maximum principle does not apply directly to this problem, these steady states appear from numerical calculations to act as a threshold for blow-up: If initial data is taken in the form

$$
u_{0}(x)=\lambda u_{s}(x)
$$

and $\lambda<1$, then the solution $u(x, t) \rightarrow 0$ as $t \rightarrow \infty$, whereas, if $\lambda>1$, then $u(x, t)$ blows up in a finite time $T$. Since the steady states $u_{s}(x)$ become unbounded as $\mu \rightarrow 1$, the threshold for blow-up also becomes unbounded; this motivates our observation that blow-up does not occur for $\mu=1$. We formally conclude from this discussion that blow-up is unlikely for the case where $\mu=1$.

Formal Proposition II (blow-up for general $\mu<1$ ). If the initial form of $u(x, 0)$ is sufficiently large and has a single positive maximum at the point $x=0$, then (a) if $0 \leq \mu<\frac{1}{2}$, the solutions of (1.1)-(1.4) blow up nonuniformly, with a pronounced peak at $x=0$. As $t \rightarrow T^{-}$,

$$
u(0, t) \approx \frac{1}{T-t}
$$

and the peak has "width"

$$
(T-t)^{1 / 2-\mu} \quad \text { for } \mu<\frac{1}{2}
$$

and "width"

$$
\frac{1}{\log (T-t)} \quad \text { for } \mu=\frac{1}{2}
$$


(b) If $\frac{1}{2}<\mu<1$, then problems (1.1)-(1.4) have solutions that blow up globally in a finite time $T$. As $t \rightarrow T^{-}$, then there is a function $U(x)$ such that, apart from $a$ boundary layer close to $x=1$ of decreasing width as $t \rightarrow T$, we have

$$
u(x, t) \approx \frac{U(x)}{(T-t)}
$$

Moreover,

$$
u(0, t) \approx \frac{1}{2(T-t)(1-\mu)}
$$

and

$$
u(1, t) \approx \frac{1-2 \mu}{2(T-t)(1-\mu)}
$$

Furthermore, as $\mu \rightarrow 1$,

$$
u(x, t) \approx \frac{1}{2} \frac{\cos (\pi x)}{(T-t)(1-\mu)} .
$$

When $\mu \geq 1$, a quite different situation arises, shown in the following theorem.

TheOREM III (decay $\mu=1$ ). (a) For $\mu=1$, problems (1.1)-(1.4) have exact decaying solutions with the form

$$
\begin{gathered}
u(x, t)=A e^{-n^{2} \pi^{2} t} \cos (n \pi x), \\
v(x, t)=A e^{-n^{2} \pi^{2} t} \sin (n \pi x) / n \pi
\end{gathered}
$$

for any $A \in \mathbb{R}$ and any integer $n$. Let

(b) The exact solution with $n=1$ is asymptotically stable in the following sense:

$$
u(x, t)=A e^{-\pi^{2} t}[\cos (\pi x)+\tilde{u}(x, t)]
$$

and assume that $\tilde{u}(x, 0) \in H^{2}(0,1)$. Then, letting $\|\bullet\|_{2}$ denote the standard $L_{2}$ norm, for any $\varepsilon>0$, there exists $\delta=\delta(A)>0$ such that

$$
\|\tilde{u}(x, 0)\|_{2} \leq \delta \Rightarrow\|\tilde{u}(x, t)\|_{2} \leq \varepsilon \quad \forall t \geq 0
$$

and, in addition,

$$
\int_{0}^{1} \tilde{u}(x, t) \cos (m \pi x) d x \rightarrow 0 \quad \text { as } t \rightarrow \infty
$$

uniformly in $m$ for all $m \geq 2$.

(c) If $\mu=4$ and $u(x, 0) \in H^{2}(0,1)$, then, for any $u_{0}$,

$$
\|u(x, t)\|_{\infty} \rightarrow 0 \quad \text { as } t \rightarrow \infty
$$

Furthermore, we present the following conjecture.

ConjeCture IV (decay $\mu \geq 1$ ). (a) For almost all initial data, there is a constant A such that, as $t \rightarrow \infty$,

$$
e^{\pi^{2} t} u(x, t) \rightarrow A \cos (\pi x)
$$

Thus, in general, the solutions of (1.1)-(1.4) decay to 0 as $t \rightarrow \infty$. 
(b) $\|u(x, t)\|_{\infty} \rightarrow 0$ as $t \rightarrow 0$ for all $\mu \geq 1$.

Conjecture IV and result (b) in Theorem III indicate that the observed decay of the solutions of the Navier-Stokes equations is preserved for $\mu>1$.

The outline of the paper is as follows. In $\S 2$ we show how problems (1.1)-(1.3) can be derived as a similarity solution of the Navier-Stokes equations when $\mu=$ 1. Previous related work on blow-up for the Navier-Stokes and Euler equations is discussed. In $\S 3$ we briefly outline an existence and regularity theory for the evolution equation, study the general evolution of system (1.1), and consider the asymptotic form of the steady-state solutions for $\mu$ near 1; our asymptotic formulae are supported by some numerical calculations of the steady state. In $\S 4$ we study the solutions that blow up as $t \rightarrow T$ and, in particular, we look at the transition from nonuniform to uniform blow-up at $\mu=\frac{1}{2}$ and the nature of the blow-up behaviour in the limit $\mu \rightarrow 1$. We compare our asymptotic calculations with some numerical calculations made using the adaptive mesh method described in [BDS1]. In $\S 5$ we study the limit of the equations corresponding to the Navier-Stokes equations $(\mu=1)$, describe the exact solutions, prove the stability result, and present the results of some numerical experiments for the case where $\mu=1$. Finally, in $\S 6$ we discuss the case of $\mu>1$.

2. Similarity solutions of the Navier-Stokes equations. The incompressible Navier-Stokes equations with velocity field $\left(u^{*}, v^{*}, w^{*}\right)$ and pressure $p^{*}$, all dependent upon $(x, y, z, t)$, posed in a channel that is infinitely long in the $x$ - and $z$-directions but finite in the $y$-direction, admit similarity solutions of the form

$$
\begin{gathered}
u^{*}=-x u(y, t), \\
v^{*}=v(y, t), \\
w^{*}=-z w(y, t), \\
p^{*}=p(y, t)-\frac{1}{2}\left(c(t) x^{2}+e(t) z^{2}\right) .
\end{gathered}
$$

Substituting this form of solution into the Navier-Stokes equations with Reynolds number $1 / \nu$, we obtain the following equations:

$$
\begin{gathered}
u_{t}-u^{2}+v u_{y}=\nu u_{y y}-c \\
v_{t}+v v_{y}=\nu v_{y y}-p_{y} \\
w_{t}-w^{2}+v w_{y}=\nu w_{y y}-e \\
v_{y}=u+w
\end{gathered}
$$

A similarity solution related to that above was used in [Stu] to construct solutions of the Euler equations in three dimensions that develop singularities in finite time. Recently, Childress et al. [CISY] made the observation that, using the same similarity structure as [Stu] restricted to two dimensions, solutions to the Euler equations that develop singularities in finite time can still be found. This is interesting for the following reason: It is known that, in two dimensions, the solutions of both the Euler and Navier-Stokes equations with smooth initial data and finite initial kinetic energy remain smooth for all time [Kat], [Tem]. Thus the singularities found by [CISY] and [Stu] may be thought of as a consequence of the unbounded nature of the initial kinetic energy that arises from the linearity of the similarity solution in $x$ and $z$; nevertheless, the possibility of singularity development in the similarity solutions remains a question of theoretical importance.

The work in [CISY] is concerned with two-dimensional flows. Consequently, a vorticity-streamfunction formulation of the problem is used with similarity form equivalent to a two-dimensional restriction of the form above. We prefer to work in the 
primitive variables so that possible extensions of the work to three dimensions are clearer. In [CISY] numerical evidence is presented to suggest that the introduction of viscosity does not arrest the singularity formation found in the Euler equations, provided that the initial data is sufficiently large. However, this evidence was by no means conclusive, and conflicting numerical results can be found in [Cox]. We further investigate viscous singularity development; however, it is important to emphasise that the boundary conditions (1.3) are different from those used in [CISY].

We seek solutions of the equations above posed in a channel corresponding to the finite interval $0<y<1$. This necessitates the imposition of boundary conditions on $u, v$, and $w$ at both $y=0$ and $y=1$. Since (2.1), (2.3), and (2.4) are fifth order in space and we have six boundary conditions, we must allow one of $c(t)$ or $e(t)$ to be an unknown function. Without loss of generality, we fix $e(t)$ and consider $c(t)$ as an unknown. Note that the pressure $p(t)$ can be determined a posteriori from (2.2) once $u, v, w$, and $c$ have been found to satisfy (2.1), (2.3), and (2.4).

For simplicity, we consider solutions with $w(y, t) \equiv 0$ and $e(t)=0$; we also assume that $\nu=1$ as solutions for general $\nu$ can be obtained by simple rescaling. It is natural to consider the case of no flow through the walls of the channel so that $v(0, t)=v(1, t)=0$. As a second boundary condition, we consider the stress-free case with $u_{y}=0$. This allows us to directly compare the solutions of our system with those studied in [BDS1]. The alternative case of the no-slip condition at the boundaries is discussed in [CISY] and [ZDB]. These considerations then lead to the following problem:

$$
\begin{gathered}
u_{t}+v u_{y}=u_{y y}+u^{2}-c, \\
v_{y}=u,
\end{gathered}
$$

and boundary conditions

$$
u_{y}(0, t)=u_{y}(1, t)=v(0, t)=v(1, t)=0 .
$$

The initial condition on $u(y, 0)$ is chosen to be compatible with (2.6), (2.7). For notational convenience, we have let $y \rightarrow x$ in the remainder of the paper as the resulting equation then more closely resembles the reaction-diffusion equations studied in [BDS1] and elsewhere. We also set $c=K^{2}$, since (1.6) shows that it is positive. Equations (2.5) $-(2.7)$ then give (1.1) $-(1.3)$ if $\mu=1$.

3. The general evolution of (1.1) and its steady state solutions. Equation (1.1), together with its initial and boundary conditions, is an example of a nonlinear parabolic equation with a constraint and a preferred convective direction. Indeed, if we consider a solution $u(x, t)$, which is positive at $x=0$ and has one zero, then $v(x, t)$ will be positive for all $x$, and hence the convection will be in the direction of increasing $x$; that is, away from the region where $u(x, t)$ is positive.

We start by considering the existence and regularity of a solution to (1.1)-(1.4) and study the stability of the trivial solution $u \equiv 0$. We will use the notation

$$
\begin{gathered}
X=\left\{u \in H^{1}(0,1): \int_{0}^{1} u(s) d s=0\right\}, \\
B=\left\{u \in H^{1}(0,1):\|u\|_{H}^{1} \leq R\right\}
\end{gathered}
$$

and $\|\bullet\|_{p}$ to denote the $L_{p}(0,1)$ norm and $\|\bullet\|_{H^{s}}$ to denote the $H^{s}(0,1)$ norm. 
TheOREM 3.1. Given any $u_{0} \in B \bigcap X$, there is a time $T=T(R)$ such that (1.1)-(1.4) have a unique solution satisfying

$$
u(\bullet, t) \in C^{1}((0, T) ; X)
$$

If $T<\infty$, then $\|u(\bullet, t)\|_{H^{1}} \rightarrow \infty$ as $t \rightarrow T_{-}$. Furthermore, for any $\tau<T$, we have

$$
\|u(\bullet, t)\|_{H^{2}} \leq \frac{C(\tau, B)}{t^{1 / 2}}, \quad t \in(0, \tau] \quad \text { if } u_{0} \notin H^{2}
$$

and

$$
\|u(\bullet, t)\|_{H^{2}} \leq C(\tau, B), \quad t \in[0, \tau], \quad \text { if } u_{0} \in H^{2} .
$$

Finally, the solution $u \equiv 0$ is stable in $H^{1}(0,1)$ in the sense that there exists $\rho>0, M \geq 1$, and $\beta>0$ such that if

$$
\left\|u_{0}(\bullet)\right\|_{H^{1}} \leq \frac{\rho}{2 M}
$$

then $T=\infty$ and

$$
\|u(\bullet, t)\|_{H^{1}} \leq 2 M e^{-\beta t}\left\|u_{0}(\bullet)\right\|
$$

Proof. Integrating (1.2) using (1.6) we obtain from (1.1)

$$
\begin{aligned}
u_{t}-u_{x x} & =u^{2}-\mu\left[\int_{0}^{1} u(s, t) d s\right] u_{x}-(1+\mu) \int_{0}^{1} u^{2}(s, t) d s \\
u_{x}(0) & =u_{x}(1)=0
\end{aligned}
$$

This equation can be considered as an abstract evolution equation

$$
u_{t}+A u=F(u)
$$

where $A=-d^{2} / d x^{2}, D(A)$ is defined in the usual way for the Laplacian with Neumann data [Tem, p. 62] and

$$
F(u)(x):=u^{2}(x)-\mu\left[\int_{0}^{x} u(s) d s\right] u_{x}(x)-(1+\mu) \int_{0}^{1} u^{2}(s) d s .
$$

It is straightforward to verify that

$$
\begin{gathered}
\|F(u)-F(v)\|_{L^{2}} \leq C(R)\|u-v\|_{H^{1}} \quad \forall u, v \in B \\
\|F(u)\|_{H^{1}} \leq C(r)\|u\|_{H^{2}} \quad \forall u:\|u\|_{H^{2}} \leq r .
\end{gathered}
$$

Application of Theorem 6.3.1 in [Paz] gives the existence of a solution

$$
u(\bullet, t) \in C^{1}\left((0, T) ; H^{1}\right) .
$$

Furthermore, integrating (1.1) then shows that since $u_{0} \in X$, we have $u(\bullet, t) \in X$ for all $t \in(0, T)$ and the existence result follows. The continuation result for bounded $\|u\|_{H^{1}}$ also follows from the application of the same theorem. 
To obtain $H^{2}(0,1)$ norm bounds, we use the variation of constants formulation for the solution of $(3.1)$ :

$$
u(\bullet, t)=e^{A t} u_{0}+\int_{0}^{t} e^{A(t-s)} F(u(\bullet, s)) d s .
$$

Taking norms and using the smoothing of the analytic semigroup $e^{A t}$ gives

$$
\begin{gathered}
\|u(\bullet, t)\|_{H^{2}} \leq\left\|e^{A t} u_{0}\right\|_{H^{2}}+\int_{0}^{t}\left\|e^{A(t-s)} F(u(s))\right\|_{H^{2}} d s \\
\Rightarrow\|u(\bullet, t)\|_{H^{2}} \leq C(\tau)\left\|u_{0}\right\|_{H^{2}}+\int_{0}^{t} \frac{1}{(t-s)^{1 / 2}}\|F(u(s))\|_{H^{1}} d s, \quad t \in(0, \tau) .
\end{gathered}
$$

Using (3.2) application of the Gronwall inequality of [Hen, p. 6] gives the desired $H^{2}(0,1)$ bound in the case of initial data in $H^{2}(0,1)$. If the initial data is in $H^{1}(0,1)$, we observe that

$$
\|u(\bullet, t)\|_{H^{2}} \leq \frac{1}{t^{1 / 2}}\left\|u_{0}\right\|_{H^{2}}+\int_{0}^{t} \frac{1}{(t-s)^{1 / 2}}\|F(u(s))\|_{H^{1}} d s
$$

and, again, application of the Gronwall inequality of [Hen, p. 6] gives the desired result.

Finally, to prove stability of the trivial solution $u \equiv 0$, it is sufficient to observe that the spectrum of $A$ subject to the integral constraint (1.5) is real, positive, and bounded from zero. Theorem 5.1.1 in [Hen] then gives the desired stability result.

Numerical experiments indicate that the resulting solution either decays to zero or blows up in a finite time and that all steady states other than the zero steady state are unstable. Indeed, for $\mu<1$, there appear to be thresholds on the initial data such that solutions that are initially below these thresholds decay to zero and those above blow up. To gain insight into these thresholds, we first study the steady state solutions.

The steady solutions of (1.1)-(1.4) satisfy the following boundary value problem: Find $u(x) \in C^{2}(0,1), v(x) \in C^{1}(0,1)$, and $K \in R$ such that

$$
\begin{gathered}
\mu u_{x} v=u_{x x}+u^{2}-K^{2}, \\
v_{x}=u, \\
u_{x}(0)=u_{x}(1)=v(0)=v(1)=0 .
\end{gathered}
$$

We now consider the existence of nonzero steady states for $\mu<1$.

3.1. Steady states. Formal Proposition I. To prove existence, we apply a rescaling so that

$$
\begin{gathered}
s=K^{1 / 2} x \\
u(x)=K w\left(K^{1 / 2} x\right), \\
v(x)=K^{1 / 2} z\left(K^{1 / 2} x\right) .
\end{gathered}
$$

Then $w(s)$ and $z(s)$ satisfy the differential equation

$$
\begin{gathered}
\mu w_{s} z=w_{s s}+w^{2}-1, \\
z_{s}=w
\end{gathered}
$$


and

$$
w_{s}(0)=w_{s}\left(K^{1 / 2}\right)=z(0)=z\left(K^{1 / 2}\right)=0
$$

so that $w(s)$ satisfies the integral constraint

$$
\int_{0}^{K^{1 / 2}} w(s) d s=0 .
$$

In [BDS1] it is shown by phase plane arguments that this system has nonzero solutions when $\mu=0$, and a simple extension of the proof using continuity arguments shows that these solutions persist for small $\mu$.

LEMMA 3.2. If $\mu$ is sufficiently small then, for each integer $n>0$ the problem (3.3)-(3.5) has precisely two nonzero solutions $u_{n}^{+}(x)$ and $u_{n}^{-}(x)$ such that $u_{n}^{+}(0)>$ $0>u_{n}^{-}(0)$ and $u_{n}^{+}$and $u_{n}^{-}$have precisely $n$ zeros in the interval $(0,1)$.

Numerical calculations using the path-following code AUTO [DOK], indicate that these steady state solutions continue to exist for all values of $\mu<1$. Moreover, as $\mu \rightarrow 1$, our numerical calculations strongly imply that there is a steady state solution $u_{1}(x)$ with one zero that becomes unbounded as $\mu \rightarrow 1$, such that

$$
(1-\mu) u_{1}(0) \rightarrow \pi^{2} / 2
$$

In Fig. 3.1 we present a graph of $u_{1}(0)$ as a function of $\mu$, and in Figs. 3.2 and 3.3 we show the form of $u_{1}(x)$ and $v_{1}(x)$ when $\mu=0.95$. We can see clearly from these figures that $u_{1}(x)$ and $v_{1}(x)$ are close to the cosine and sine functions, respectively. We now derive a formal asymptotic explanation of this phenomenon.

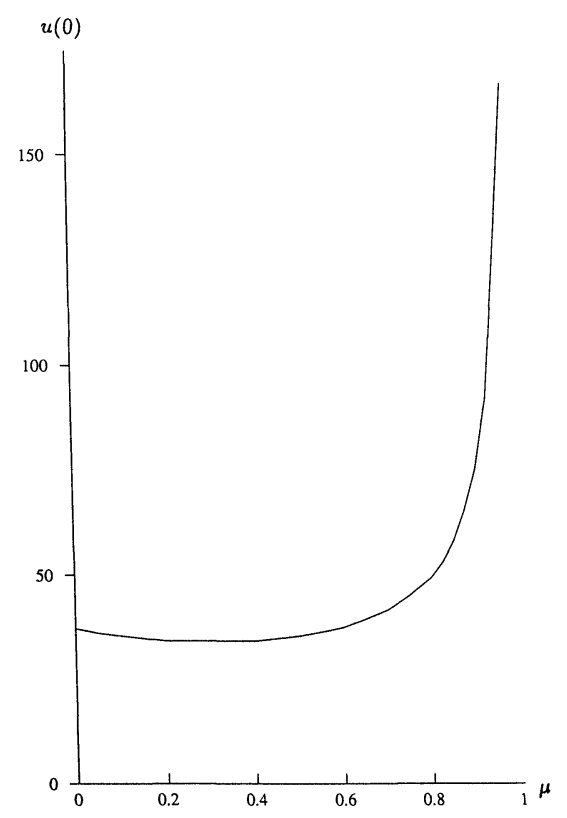

FIG. 3.1. The variation of $u(0)$ as a function of $\mu$. 


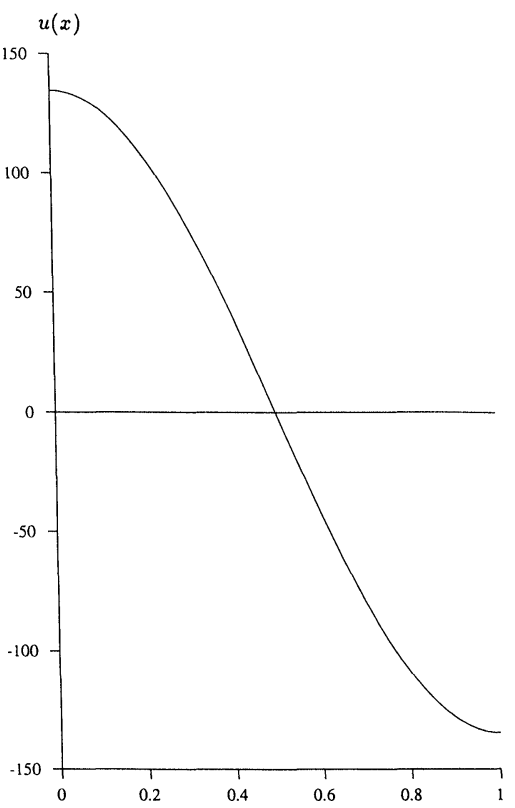

FIG. 3.2. The function $u(x)$ when $\mu=0.95$.

3.2. Asymptotic form of the steady state for $\boldsymbol{\mu} \approx 1$. Motivated by the numerical calculations described above, we set $\varepsilon=1-\mu$ and

$$
u=\varepsilon^{-1} \sum_{i=0}^{\infty} \varepsilon^{i} u_{i}, \quad v=\varepsilon^{-1} \sum_{i=0}^{\infty} \varepsilon^{i} v_{i}, \quad K^{2}=\varepsilon^{-2} \sum_{i=0}^{\infty} \varepsilon^{i} K_{i} .
$$

In this subsection all subscripts for $u$ and $v$ refer to these asymptotic expressions and should not be confused with their usage in Lemma 3.1 where $n$ denotes the number of zeros. We seek solutions with one zero in $(0,1)$; other solutions with more zeros may be obtained from this solution by extension, reflexion, and rescaling. In particular, if $u(x)$ is a solution with one zero on the interval $[0,1]$ that we extend by sucessive reflexions in the lines $x=k$ for integer $k$, then a solution $U(x)$ with $n$ zeros is given simply by

$$
U(x)=n^{2} u(n x) .
$$

Substituting into (3.3)-(3.5) we find that, to leading order,

$$
\begin{gathered}
v_{0} u_{0}^{\prime}-u_{0}^{2}+K_{0}=0, \\
v_{0}^{\prime}=u_{0} .
\end{gathered}
$$

The problem is of a singular perturbation type and the leading order problem is of lower order than the original problem. Differentiating (3.13) and eliminating $u_{0}$ gives

$$
v_{0} v_{0}^{\prime \prime \prime}-v_{0}^{\prime} v_{0}^{\prime \prime}=0 .
$$

This is the Wronskian of $v_{0}$ and $v_{0}^{\prime \prime}$ and hence has solutions of the form

$$
v_{0}=a \sin (b x+c)
$$




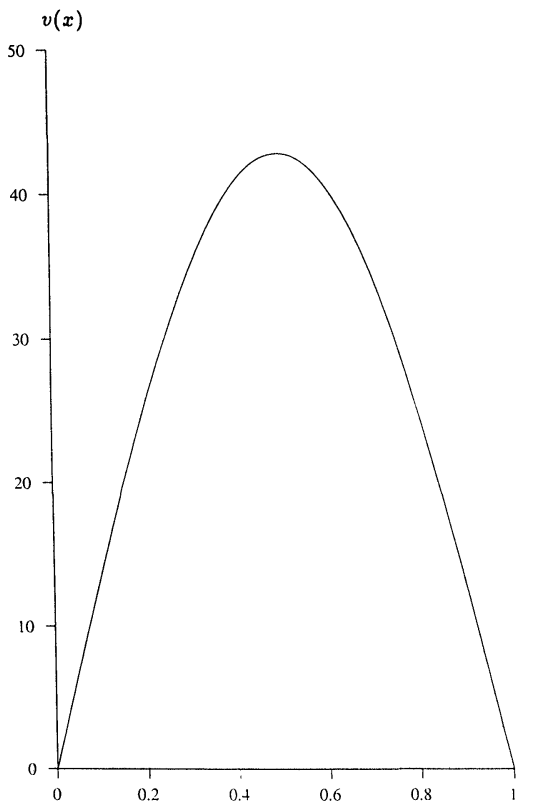

FIG. 3.3. The function $v(x)$ when $\mu=0.95$.

for arbitrary $a, b, c \in \mathbb{R}$. Setting $c=0$ and $b=\pi$, we obtain a solution of the leading order problem for which $u_{0}(x)$ has one zero and which satisfies the boundary conditions. It is significant that at this level of the asymptotic approximation, the leading order solution has an undetermined amplitude $a$. Thus it has the form

$$
v_{0}(x)=a \sin (\pi x), \quad K_{0}=a^{2} \pi^{2}, \quad u_{0}(x)=a \pi \cos (\pi x) .
$$

This is an unusual situation in singular perturbation theory since we have found leading order solutions that satisfy all the boundary conditions and, furthermore, have arbitrary amplitude $a$.

Proceeding to the next order, we obtain the equations

$$
v_{1} u_{0}^{\prime}+u_{1}^{\prime} v_{0}-2 u_{1} u_{0}+K_{1}=v_{0} u_{0}^{\prime}+u_{0}^{\prime \prime} \quad \text { and } \quad v_{1}^{\prime}=u_{1} .
$$

Substituting the above expression for $u_{0}(x)$ and eliminating $u_{1}$ gives the differential equation

$$
\begin{gathered}
a \sin (\pi x) v_{1}^{\prime \prime}-2 a \pi \cos (\pi x) v_{1}^{\prime}-a \pi^{2} \sin (\pi x) v_{1}+K_{1} \\
=-a^{2} \pi^{2} \sin ^{2}(\pi x)-a \pi^{3} \cos (\pi x)
\end{gathered}
$$

or

$$
L v_{1}=-a \pi^{2} \sin ^{2}(\pi x)-\pi^{3} \cos (\pi x)-K_{1} / a,
$$

where

$$
L \psi:=\sin (\pi x) \psi^{\prime \prime}-2 \pi \cos (\pi x) \psi^{\prime}-\pi^{2} \sin (\pi x) \psi
$$


We now study the properties of the operator $L$ when $x$ is small so that we are close to the boundary $x=0$. We find that

$$
L \psi \approx\left(\pi x-\frac{\pi^{3} x^{3}}{6}\right) \psi^{\prime \prime}-2 \pi\left(1-\frac{\pi^{2} x^{2}}{2}\right) \psi^{\prime}-\pi^{2}\left(\pi x-\frac{\pi^{3} x^{3}}{6}\right) \psi .
$$

Because $v_{1}(x)$ is an odd function and $v_{1}(0)=0$, we postulate the existence of a regular solution to (3.15) of the form

$$
v_{1}(x)=c_{0} x+c_{1} x^{3}+c_{2} x^{5}+\cdots .
$$

In this case,

$$
L v_{1}=-2 \pi c_{0}+\left(6 \pi c_{1}-6 \pi c_{1}+\pi^{3} c_{0}-\pi^{3} c_{0}\right) x^{2}+\mathcal{O}\left(x^{4}\right)=-2 \pi c_{0}+\mathcal{O}\left(x^{4}\right) .
$$

We conclude that the function $x^{2}$ is not in the range of the operator $L$ when $L$ is applied to a regular function of the form above. Thus, to find a regular solution of (3.15) in a neighbourhood of $x=0$, we require that the right-hand side of this expression has no contribution of $\mathcal{O}\left(x^{2}\right)$. This implies that

$$
-a^{2} \pi^{4}+a \pi^{5} / 2=0
$$

and hence that

$$
a=\pi / 2 .
$$

Thus we obtain a solution to (3.3)-(3.6) that is regular at $x=0$ only if

$$
\begin{aligned}
& v_{0}(x)=\frac{\pi}{2} \sin (\pi x), \\
& u_{0}(x)=\frac{\pi^{2}}{2} \cos (\pi x),
\end{aligned}
$$

and

$$
K_{0}=\frac{\pi^{4}}{4}
$$

We note that this value of $a$ implies that $u_{0}(x)$ has a positive maximum at $x=0$. (To obtain leading order solutions with $n$ zeros, we simply rescale these expressions to give, for example, $u_{0}(x)=n^{2} \pi^{2} / 2 \cos (n \pi x)$.)

The leading order solution is in very good agreement with the numerical calculations presented in Fig. 3.2. In particular, we have correctly predicted the value of the constant $a$ that appeared to be arbitrary at the leading order of the asymptotic expansion. This prediction of the value of $a$ follows directly from our assumption of the regularity of the functions $u_{1}(x)$ and $v_{1}(x)$ at the point $x=0$.

To further investigate our assumption of regularity, we now study the solution of (3.15) in the neighbourhood of the boundary $x=1$. Accordingly, we set $y=1-x$ and consider $u_{0}$ and $v_{0}$ to be functions of $y$ so that

$$
v_{0}(y)=\frac{\pi}{2} \sin (\pi(1-y))=\frac{\pi}{2} \sin (\pi y)
$$

and

$$
u_{0}(y)=\frac{\pi^{2}}{2} \cos (\pi(1-y))=-\frac{\pi^{2}}{2} \cos (\pi y)
$$


Denoting differentiation with respect to $y$ by ', we obtain from (3.15)

$$
\begin{gathered}
\frac{\pi}{2} \sin (\pi y) v_{1}^{\prime \prime}-\pi^{2} \cos (\pi y) v_{1}^{\prime}-\frac{\pi^{3}}{2} \sin (\pi y) v_{1}+K_{1} \\
=-\frac{\pi^{4}}{4} \sin ^{2}(\pi y)+\frac{\pi^{4}}{2} \cos (\pi y) .
\end{gathered}
$$

For small $y$, we obtain

$$
L v_{1}=-\frac{\pi^{3}}{2} \sin ^{2}(\pi y)+\pi^{3} \cos (\pi y)+2 \frac{K_{1}}{\pi}=\pi^{3}+\frac{2 K_{1}}{\pi}-\pi^{5} y^{2}+\mathcal{O}\left(y^{4}\right)
$$

for $L$ as defined earlier. We now see that the range of the operator $L$ has a term of the form $-\pi^{5} y^{2}$. Thus, in view of the discussion above, we cannot construct a regular expansion for $v_{1}(y)$ for which $v_{1}=0$ when $y=0$. Accordingly for small $y$, we must pose instead a singular expansion for $v_{1}(y)$, taking the form

$$
v_{1}(y)=A y+B y^{3}+C y^{3} \log (y)+\mathcal{O}\left(y^{5} \log (y)\right) .
$$

$K_{1}$ is undetermined at this stage of the calculation and the constants $A$ and $B$ may take arbitrary values. However, the value of the constant $C$ is determined by expression (3.16) and takes the value

$$
C=-\frac{\pi^{4}}{3}
$$

Thus for small $y$,

$$
v_{1}(y)=A y+B y^{3}-\frac{\pi^{4}}{3} y^{3} \log (y)+\cdots
$$

Differentiating this expression, we see that $v_{1}^{\prime \prime \prime}=\mathcal{O}\left(\pi^{4} \log (y)\right)$. Consequently, this expression is singular for $y=0$, and hence is not a valid approximation for $v_{1}(y)$ for very small $y$. Instead, it is the limit as $y \rightarrow 0$ of an outer expression for the solution of the original differential equation. To complete our calculation, we must evaluate this solution in a boundary layer for $y$ close to 0 and then match this with the outer solution above. This matching is possible, but the details of the calculation are somewhat technical and we do not present them here; further details are given in [BDS2]. As a consequence of this calculation, we find that the boundary layer has width proportional to $(1-\mu)^{1 / 2}$ and

$$
v_{1}^{\prime \prime \prime}(0)=-\pi^{4} \log (\varepsilon)+\mathcal{O}(1)
$$

so that $v_{1}^{\prime \prime \prime}(0) \rightarrow \infty$ as $\varepsilon \rightarrow 0$.

Combining these results gives the formulae presented in Formal Proposition I.

It is important to ask why we should impose regularity of the asymptotic expansion at $x=0$ when this is not possible at $x=1$. In fact, to be precise, the asymptotic expansion is taken to be regular at the boundary point at which $u$ is positive; indeed, the positivity of $a$ and hence of $u(0)$ is forced by the assumption of regularity. This is entirely consistent with the fact that the direction of the convection in (1.1) is from the region where $u(x, t)$ is positive to the region where it is negative. Thus, compared with other convective systems, we would expect more subtle behaviour in the function $u(x, t)$ to occur at the boundary toward which the convective flow is directed and 
hence at the boundary at which $u(x, t)$ is negative. We note that if we impose regularity on $v_{1}(x)$ at the point $x=1$ rather than $x=0$, we simply obtain a reflexion of the earlier solution with $u(x)$ positive at $x=1$. If we were to drop all the assumptions of regularity so that $v_{1}(x)$ is neither regular at the point $x=0$ nor at the point $x=1$, then the value of the constant $a$ is no longer determined uniquely, and a boundary layer must be introduced at both points. However, the calculations reported in [BDS2] indicate that it is not possible to find a consistent matched asymptotic solution for $u(x)$ in this case.

3.3. Numerical results. We conclude this section with some numerical calculations. Using the package AUTO [DOK], we may compute the functions $u(x)$ and $v(x)$ for values of $\mu$ very close to 1 . In Figs. 3.2 and 3.3, we presented $u(x)$ and $v(x)$ for $\mu=0.95$. In Figs. 3.4 and 3.5, we present numerical calculations of the functions $u_{1}(x)$ and $u_{1}^{\prime}(x)$ for $\mu=0.9995$. In these figures the regular form of the functions at $x=0$ and the boundary layer at $x=1$ can be seen clearly. In Fig. 3.6, we present a graph of $u_{1}^{\prime \prime}(1)=v_{1}^{\prime \prime \prime}(1)$ as a function of $\log (1-\mu)$. It is apparent that the points on this graph fall on a straight line in accordance with (3.17), and an estimate of the slope and intercept of this line gives

$$
u_{1}^{\prime \prime}(1)=v_{1}^{\prime \prime \prime}(1) \approx 97.15 \log (1-\mu)+224.13
$$

We can compare this directly with the asymptotic estimate for $v_{1}^{\prime \prime \prime}$, and we find that the calculation gives a slope very close to the predicted value of $\pi^{4}$.

4. Blow-up results. Formal Proposition II. In this section we establish the Formal Proposition II. As we have observed from numerical experiments, it appears that the threshold separating initial data that leads to solutions that blow up from that leading to solutions that decay to zero is closely related to the steady state solutions constructed in $\S 3$, and becomes unbounded as $\mu \rightarrow 1$. This strongly implies that blow-up ceases to occur when $\mu=1$. In this section we examine in more detail, solutions that blow up in time in the limit as $\mu \rightarrow 1$, as well as study the general case of $\mu<1$.

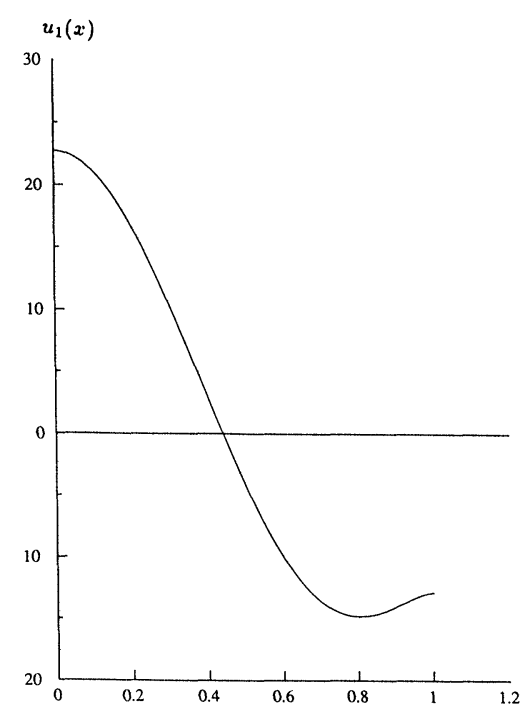

FIG. 3.4. The function $u_{1}(x)$ when $\mu=0.9995$. 


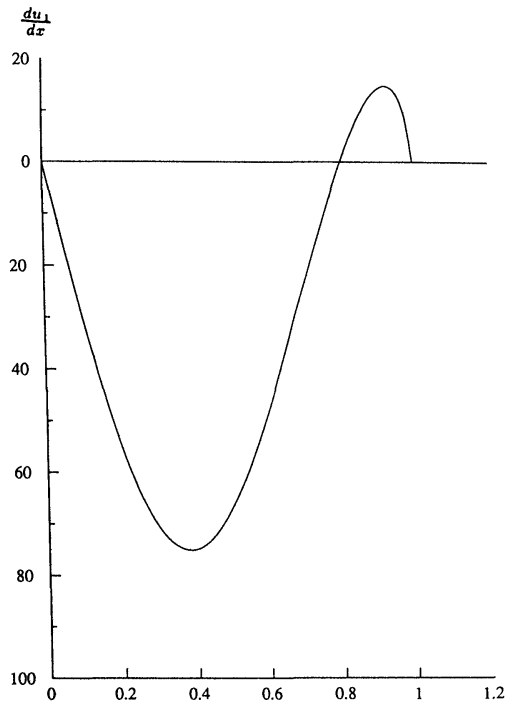

FIG. 3.5. The function $d u_{1} / d x$ when $\mu=0.9995$.

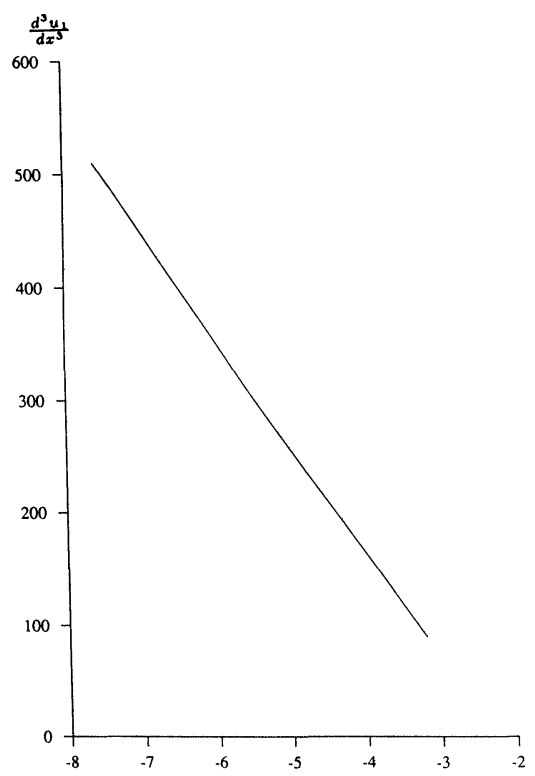

FIG. 3.6. $d^{3} u_{1} / d x^{3}$ when $x=1$ plotted as a function of $\log (1-\mu)$.

In Figs. 4.1 and 4.2, we present numerically computed profiles of two solutions that blow up for $\mu=0.25$ and $\mu=0.75$. In each case, the initial data has the form $u(x, 0)=100 \cos (\pi x)$ and $u(0,0)$ is larger than the value taken by the steady state solution. (These solutions were computed using a variation of the adaptive mesh algorithm described in [BDS2].) We observe from these two figures that there is a marked contrast between the form of blow-up in these two cases. In the first case, the function $u(x, t)$ develops a pronounced spike close to $x=0$ and the blow-up is most rapid at this point. In contrast, when $\mu=0.75$, the function $u(x, t)$ appears 


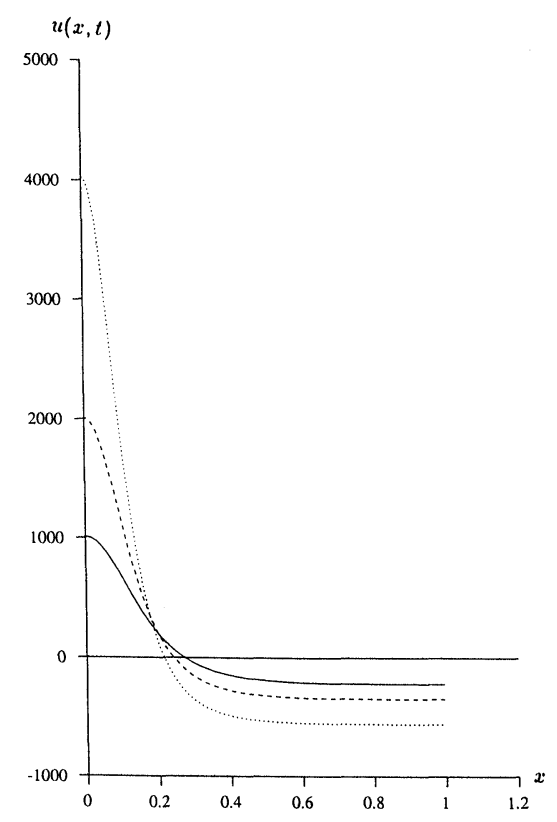

FIG. 4.1. The nonuniform blow-up of $u(x, t)$ when $\mu=0.25$.

to blow up at a uniform rate throughout the interval $[0,1]$. More detailed numerical experiments reveal that the transition between the nonuniform and uniform forms of blow-up occurs when $\mu=\frac{1}{2}$. For $\mu \leq \frac{1}{2}$, the blow-up is similar in form to that described for $\mu=0$ in [BDS2] whereas, if $\frac{1}{2}<\mu<1$, it takes on the uniformly global character.

We now show how the value of $\mu$ affects the form of blow-up. We look at the two cases $0<\mu \leq \frac{1}{2}, \frac{1}{2}<\mu<1$ separately, but for each discussion we assume that blow-up is such that the function $u(x, t)$ has a single postive maximum at the origin. This can always be assumed for appropriate initial data and, generically, we would always expect such a profile (or a reflexion of such).

4.1. Nonuniform blow-up: $0<\boldsymbol{\mu} \leq \frac{1}{2}$. Motivated by the scalings introduced in [BDS2], we presume that blow-up occurs at the origin and that $u(x, t)$ has a similarity solution structure characterised by the following change of variables.

$$
u=\frac{U(\eta, \xi)}{T-t}, \quad \xi=-\ln (T-t), \quad \eta=x /(T-t)^{\theta}=x e^{\theta \xi}
$$

Here we assume that in the blow-up region, $\eta$ is an $\mathcal{O}(1)$ quantity and that the expression relating $x$ to $\eta$ gives a scaling for the blow-up region that tends to zero as $t \rightarrow T$. The variable $\xi$ can be thought of as a rescaled time so that $\xi \rightarrow \infty$ as the blow-up time $T$ is approached. The value of the exponent $\theta$ is undetermined at this stage. Under this transformation, (1.1) becomes

$$
U_{\xi}+\theta \eta U_{\eta}+\mu U_{\eta} \int_{0}^{\eta} U d \eta=U^{2}-U+e^{(2 \theta-1) \xi} U_{\eta \eta}-(1+\mu) e^{-\theta \xi} \int_{0}^{e^{\theta \xi}} U^{2} d \eta
$$

We now take the limit $\xi \rightarrow \infty$ of this expression to determine the profile of $u$ close to the blow-up time. To do this, we must assume that $0<\theta<\frac{1}{2}$ and we presume 


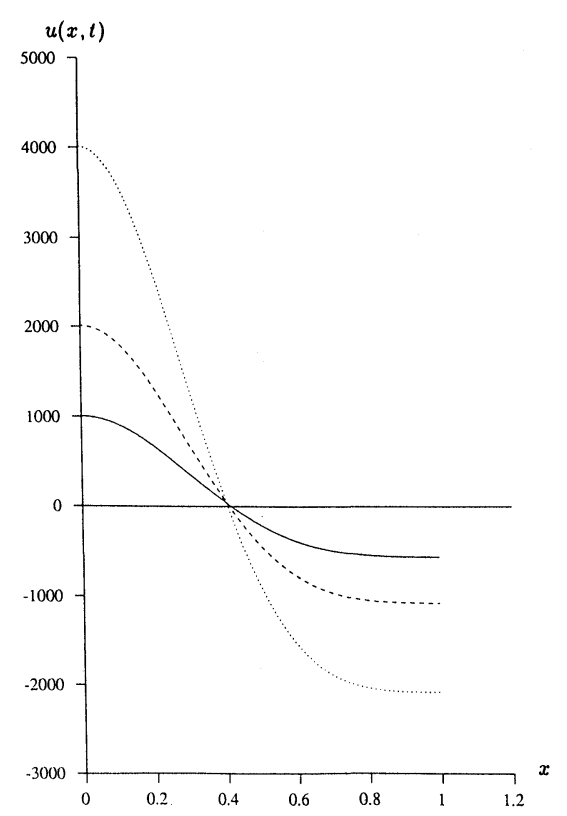

FIG. 4.2. The uniform blow-up of $u(x, t)$ when $\mu=0.75$.

further that, in this limit, $U \sim U(\eta)$. Following the discussions in $\S 3$ we also assume that $U(\eta)$ is a regular function of $\eta$ for $\eta$ close to 0 . It is further convenient to set $\alpha=\theta+\mu$, which then gives the following first order differential equation for $U(\eta)$ :

$$
\left[(\alpha-\mu) \eta+\mu \int_{0}^{\eta} U d \eta\right] U^{\prime}=U^{2}-U
$$

We note that the equation is invariant if we replace $\eta$ by $A \eta$ for any constant $A \neq 0$. Thus if $U(\eta)$ is a solution, then so is $U(A \eta)$ for any $A$.

Following our assumption of the regularity of $U(\eta)$, we now consider a series solution for $U(\eta)$ of the form

$$
U=a_{o}+\sum_{i=1}^{\infty} a_{i} \eta^{2 i}
$$

in an attempt to further elucidate the structure of blow-up. Substituting this expression into the differential equation leads to the following conditions upon the coefficients:

$$
\begin{aligned}
a_{o}\left(1-a_{o}\right) & =0 \\
a_{1}(2 \alpha-1) & =0 \\
a_{2}(4 \alpha-1) & =a_{1} a_{1}\left[1-\mu \frac{2}{3}\right], \\
a_{3}(6 \alpha-1) & =a_{1} a_{2}\left[2-\mu\left(\frac{4}{3}+\frac{2}{5}\right)\right],
\end{aligned}
$$




$$
a_{4}(8 \alpha-1)=a_{1} a_{3}\left[2-\mu\left(\frac{6}{3}+\frac{2}{7}\right)\right]+a_{2} a_{2}\left[1-\mu \frac{4}{5}\right],
$$

so that the general recurrence relation for $i>1$ is

$$
a_{i}(2 i \alpha-1)=\sum_{j=1}^{i-1} a_{j} a_{i-j}\left(1-\mu \frac{2 j}{2(i-j)+1}\right)
$$

Hence, exploiting the normalisation implied by the scaling invariance above, we find that $a_{o}=1$, so that

$$
u(0, t)=\frac{1}{(T-t)}
$$

Moreover, it holds that

$$
\alpha=\frac{1}{2}, \quad a_{1}= \pm 1
$$

or

$$
\alpha=\frac{1}{4}, \quad a_{1}=0, \quad a_{2}= \pm 1
$$

or

$$
\alpha=\frac{1}{6}, \quad a_{1}=a_{2}=0, \quad a_{3}= \pm 1
$$

with similar expressions for other values of $\alpha$ and $a_{i}$.

The blow-up structure is such that generally $a_{1} \neq 0$ and this case corresponds to all the numerical observations; from these calculations we conjecture that we expect that the case $a_{1}=0$ will be observed only for a set of initial data with measure zero and are hence unstable. Therefore, we deduce that, generically, $\alpha=\frac{1}{2}$ so that

$$
\theta=\frac{1}{2}-\mu
$$

implying that the blow-up peak has width

$$
x=\mathcal{O}\left((T-t)^{1 / 2-\mu}\right) .
$$

This decreases to zero as $t \rightarrow T$ demonstrating the nonuniform nature of the blow-up. The assumption that $\theta<\frac{1}{2}$, which was made in deriving the form of the asymptotic solution, further requires that $0<\mu<\frac{1}{2}$. To determine the general behaviour of the solution that blows up, we represent $u(x, t)$ as

$$
u=U(\eta, \xi)+p(t)+q(x, t) .
$$

The analysis of $u(x, t)$ then proceeds completely analogously to the case $\mu=0$ presented in [BDS2]. When $\mu=\frac{1}{2}$ it follows that $\theta=0$ and, instead, we introduce the transformation

$$
u=\frac{U(\eta, \xi)}{T-t}, \quad \xi=-\ln (T-t), \quad \eta=x \phi(\xi) .
$$

The analysis of this case then proceeds as above. 
4.2. Global blow-up: $\frac{1}{2}<\boldsymbol{\mu}<1$. If we now take $\mu>\frac{1}{2}$, the width of the blowup peak determined from the above formula tends to infinity as $t \rightarrow T$. This implies strongly that the blow-up region is now the whole interval $[0,1]$ and, consequently, that the blow-up is uniform in character. To study this case we consider a similar expression to $u$ as before, but we replace the scaled variable $\eta$ with the unscaled variable $x$. We find the effect of diffusion negligible in this regime apart from a thin boundary layer close to boundary at which $u(x, t)$ is negative. Away from this boundary layer the resulting analysis is similar to that for the Euler equations considered in [CISY] for $\mu=1$.

Accordingly, we introduce the transformation

$$
u=\frac{U(x, \xi)}{T-t}, \quad \xi=-\ln (T-t)
$$

which, on substitution in (1.1), implies that

$$
U_{\xi}+\mu U_{x} \int_{0}^{x} U d x=U^{2}-U-(1+\mu) \int_{0}^{1} U^{2} d x+e^{-\xi} U_{x x}
$$

Again, we take the limit $\xi \rightarrow \infty$ to give $U \sim U(x)$. The resulting expression for $U(x)$ is then

$$
\mu U^{\prime} \int_{0}^{x} U d x=U^{2}-U-(1+\mu) \int_{0}^{1} U^{2} d x=(U-\alpha)(U-\beta)
$$

after supposing that

$$
\sup U=\alpha>0 \quad \text { and } \quad \inf U=\beta<0 .
$$

It follows immediately from this equation that

$$
\alpha+\beta=1
$$

We assume, as before, that $U(x)$ is regular at the position of a positive maximum at $x=0$, so that

$$
U=\alpha+\delta x^{2}+\ldots
$$

and $\delta \neq 0$, then, equating the above at $\mathcal{O}\left(x^{2}\right)$,

$$
1+2 \alpha \mu=2 \alpha \Rightarrow \alpha=\frac{\frac{1}{2}}{1-\mu}
$$

and hence

$$
\beta=\frac{\frac{1}{2}-\mu}{1-\mu}<0
$$

We note that $\beta$ is only negative (as required) if $\frac{1}{2}<\mu<1$, complementing the range $0<\mu<\frac{1}{2}$ in which nonuniform blow-up is found. These results are in very good agreement with numerical calculations of $U(x)$. If we now set

$$
U(x)=\beta+c(1-x)^{\theta}+\ldots
$$


for $x$ close to 1 , then we may calculate $\theta$ explicitly to give

$$
\theta=\frac{1}{\mu-\frac{1}{2}} .
$$

Thus, apart from values of $\mu$ of the form $\frac{1}{2}+\frac{1}{m}, U(x)$ is not regular at the point $x=1$ where it is negative, and as $\mu \rightarrow \frac{1}{2}$ it becomes very flat. However, it follows from the discussion in $\S 3$ that the original solution $u(x, t)$ is regular at both $x=0$ and at $x=1$. The expression $u(x, t)=U(x) /(T-t)$ is, in fact the outer solution of a matched asymptotic expression with a time dependent boundary layer at $x=1$. The reduced regularity of the outer solution is reminiscent of the steady state and is a further consequence of the convection in the equation.

Following the techniques described in [CISY], we may integrate the first order differential equation for $U$ explicitly to give

$$
U^{\prime}=\frac{1}{A}(\alpha-U)^{1 / 2}(U-\beta)^{3 / 2-\mu}
$$

or

$$
x=A \int_{U}^{\alpha}(\alpha-U)^{-1 / 2}(U-\beta)^{\mu-3 / 2} d U,
$$

where $x=0$ is the position of a maximum. A simple translation gives the solution if $x=0$ is not the position of a maximum. The value of $A$ is given by the condition that if $U(x)$ has $n$ zeros, then

$$
\frac{1}{n}=A_{n} \int_{\beta}^{\alpha}(\alpha-U)^{-1 / 2}(U-\beta)^{\mu-3 / 2},
$$

which implies simply that

$$
A_{n}=\frac{A_{1}}{n}
$$

so that we may quite generally restrict ourselves to the case $n=1$. We also require that

$$
0=\int_{0}^{1} U d x=\int_{\beta}^{\alpha} U(\alpha-U)^{-1 / 2}(U-\beta)^{\mu-3 / 2} d U
$$

and

$$
\frac{|\alpha \beta|}{1+\mu}=\int_{0}^{1} U^{2} d x=A_{n} \int_{\beta}^{\alpha} U^{2}(\alpha-U)^{-1 / 2}(U-\beta)^{\mu-3 / 2} d U .
$$

However, both of the latter conditions are automatically satisfied.

For general $\mu$ we may express $x$ in terms of an incomplete Beta function of $U$. However, in the two limits $\mu \rightarrow 1$ and $\mu=\frac{1}{2}$, we may obtain a simpler expression for $x$.

We now consider the limit of the Navier-Stokes equations so that $\mu \rightarrow 1$ and we set $(1-\mu) U(x)=C(x)$. Substituting this and the previously calculated values for $\alpha$ and $\beta$ gives $\mathrm{c}$

$$
x=A_{1} \int_{C}^{1 / 2}\left(\frac{1}{2}-C\right)^{-1 / 2}\left(C+\mu-\frac{1}{2}\right)^{\mu-3 / 2} d C(1-\mu)^{(1-\mu)} .
$$


In the limit of $\mu \rightarrow 1$, this expression may be integrated exactly to give

$$
x=A_{1} \arccos (2 C)+K,
$$

and on substituting the boundary conditions to determine $A_{1}$ and $K$, we see that

$$
C=\frac{1}{2} \cos (\pi x), \quad U=\frac{1}{2(1-\mu)} \cos (\pi x) .
$$

We compare this with the estimate for the steady state of $u(x, t)$ close to $\mu=1$ calculated in $\S 3$ and see that both scale in the same way as $\mu \rightarrow 1$. This is further evidence for the absence of blow-up in the Navier-Stokes limit as initial data must become infinitely large for the function $u(x, t)$ to continue to grow and to ultimately blow up. It is interesting that the function $U(x)$ becomes more regular at $x=1$ as $\mu \rightarrow 1$.

In the alternative limit of $\mu=\frac{1}{2}$, we have $\alpha=1, \beta=0$, and

$$
x=A_{1} \int_{U}^{1} \frac{d U}{U(1-U)^{1 / 2}}
$$

which may be integrated to give

$$
U=\operatorname{sech}^{2}\left(\frac{x}{A_{1}}\right)
$$

This satisfies the condition that $U^{\prime}(0)=0$; however, the further condition that $U^{\prime}(1)=$ 0 is only satisfied if $A_{1}=0$. Crudely, this would imply that $U(x)$ takes the form of a single isolated peak at $x=0$ and is zero elsewhere. Numerical calculations indeed show that if $\mu$ is slightly greater than $\frac{1}{2}$, then $U(x)$ has a narrow peak close to $x=0$ and is nearly zero elsewhere.

4.3. Numerical results. To confirm the above asymptotic predictions we have made a scries of numcrical calculations for solutions that blow up. In particular, we calculate a solution $u(x, t)$ of the parabolic equation and determine the quantity

$$
\gamma(t)=\frac{u(0, t)^{2}}{u_{t}(0, t)}
$$

By using the analysis presented in $\S \S 4.1$ and 4.2 , we may determine $\gamma(t)$ in the two cases $\mu<\frac{1}{2}$ and $\mu>\frac{1}{2}$. This analysis implies that

$$
\mu<\frac{1}{2} \Rightarrow \gamma(t) \rightarrow 1 \quad \text { as } t \rightarrow T
$$

and

$$
\mu>\frac{1}{2} \Rightarrow \gamma(t) \rightarrow \frac{1}{2(1-\mu)} \quad \text { as } t \rightarrow T
$$

The value of $\gamma(t)$ as a function of $\log (u(0, t))$ is presented in Figs. 4.3 and 4.4 for $\mu=0.25,0.75$, where we have used the same initial conditions as in Figs. 4.1 and 4.2. In the two cases, $\gamma(t)$ tends to the respective limits of 1 and 2 predicted by the above formulac. 


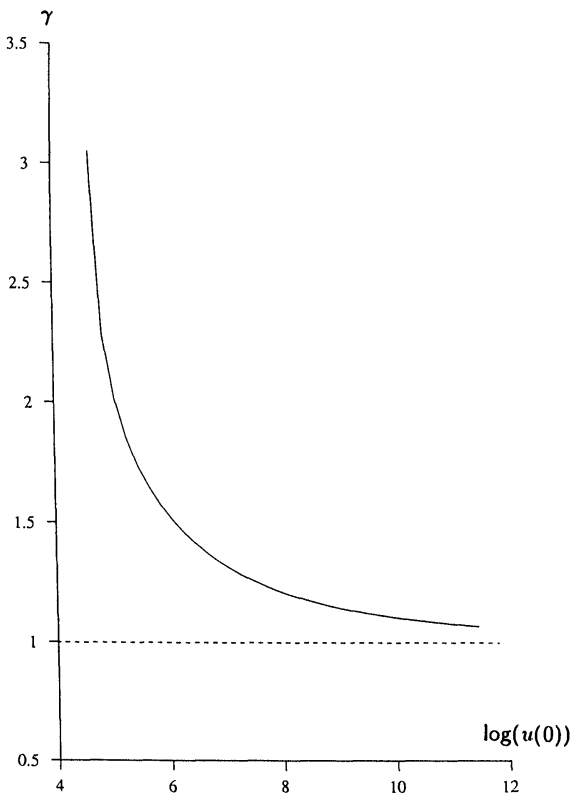

FIG. 4.3. The function $\gamma$ plotted against $\log (u(0))$ when $\mu=0.25$.

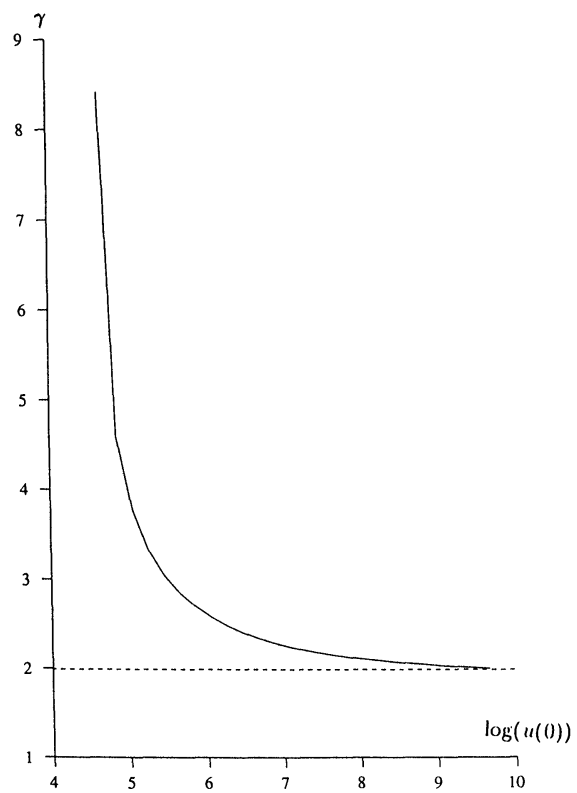

FIG. 4.4. The function $\gamma$ plotted against $\log (u(0))$ when $\mu=0.75$.

5. Behaviour for $\boldsymbol{\mu}=1$. Theorem III. In this section we complete our discussions by investigating the Navier-Stokes limit of the time dependent behaviour of (1.1)-(1.3) by taking $\mu=1$ so that the equations become, using (1.6),

$$
u_{t}+v u_{x}=u_{x x}+u^{2}-2 \int_{0}^{1} u^{2} d x, \quad 0<x<1, \quad 0<t<T,
$$




$$
v_{x}=u, \quad 0<x<1, \quad 0<t<T
$$

subject to (1.3) and appropriate initial data of zero mean. In particular, we prove Theorem III stated in $\S 1$ and show that there are decaying solutions of arbitrary norm and that in a certain sense these are attractors. This combines with our previous observations to lead us to the conjecture that the solutions of (1.1)-(1.3) do not blow up in this case. Throughout the following, the norms are as defined in $\S 3$.

The first interesting observation about this problem is that these nonlinear equations admit exact solutions; see Theorem III (a) in $\S 1$. Straightforward substitution verifies that they are solutions of the evolution equation.

These solutions are precisely those described in [Tay] transformed through the similarity variables described in $\S 2$. The exact solution with $n=1$ is asymptotically stable for arbitrary $A \in \mathbb{R}$. Since the basic solution is time dependent, the stablility result is modulo small perturbations in the direction $\cos (\pi x)$-these correspond to modifying $A$. (This is analogous to the situation in orbital stability for ordinary differential equations or stability of traveling waves in PDEs.) The precise stability result is stated in Theorem III (b) in $\S 1$.

Motivated by Theorem III (b) and also by extensive numerical computations, we make the conjecture that solutions of the form

$$
u(x, t)=A e^{-\pi^{2} t} \cos (\pi x)
$$

are attractors for the solutions resulting from almost all initial data.

We establish Theorem III (b) through a series of lemmas. First we establish that $\|\tilde{u}\|_{2}$ and $\left\|\tilde{u}_{x}\right\|_{2}$ can only grow at an exponential rate slower than $e^{\pi^{2} t}$ (Lemmas 5.3 and 5.4). We then establish that, in fact, $\|\tilde{u}\|_{2}$ is uniformly bounded for all $t$ in terms of sufficiently small initial data in $L_{2}$ (Lemma 5.5), and finally we show that all but the first Fourier cosine component $(m=1)$ decay to zero (Lemma 5.6).

We start by transforming (1.1)-(1.3) as prescribed in the theorem. We introduce the variable $\tilde{u}(x, t)$ as in the statement of the theorem and also $\tilde{v}(x, t)$ defined by

$$
v(x, t)=A e^{-\pi^{2} t}\left[\frac{\sin (\pi x)}{\pi}+\tilde{v}(x, t)\right] .
$$

In the new variables we find, for $0<x<1$,

$$
\begin{gathered}
\tilde{u}_{t}-\pi^{2} \tilde{u}+A e^{-\pi^{2} t}\left[\frac{\sin (\pi x)}{\pi} \tilde{u}_{x}-\pi \sin (\pi x) \tilde{v}+\tilde{v} \tilde{u}_{x}\right] \\
=\tilde{u}_{x x}+A e^{-\pi^{2} t}\left[2 \cos (\pi x) \tilde{u}+\tilde{u}^{2}-2 \int_{0}^{1}\left(2 \cos (\pi x) \tilde{u}+\tilde{u}^{2}\right) d x\right], \\
\tilde{v}_{x}=\tilde{u},
\end{gathered}
$$

and the boundary conditions

$$
\tilde{u}_{x}=\tilde{v}=0 \quad \text { for } \quad x=0,1 .
$$

It is a straightforward consequence of (1.5) that

$$
\int_{0}^{1} \tilde{u}(x, t) d x=0
$$

given the appropriate initial data satisfying this condition. 
Lemma 5.1. Assume that $\tilde{u}_{0} \in H^{2}(0,1)$. Given any $0<\gamma<1$, there exists $C$ independent of $A$, such that if

$$
\|\tilde{u}(x, 0)\|_{2} \leq e^{-3 A / \pi^{2}} 2 C \gamma / 3 A
$$

and if (5.3)-(5.5) have a solution for all $t \geq 0$, then

$$
\|\tilde{u}(x, t)\|_{2} \leq e^{\gamma \pi^{2} t} 2 C \gamma / 3 A \quad \forall t \geq 0 .
$$

Proof. Taking the inner product of (5.3) with $\tilde{u}$ gives

$$
\begin{aligned}
\frac{1}{2} \frac{d}{d t}\|\tilde{u}\|_{2}^{2}-\pi^{2}\|\tilde{u}\|_{2}^{2} & \\
=-\left\|\tilde{u}_{x}\right\|_{2}^{2}+A e^{-\pi^{2} t}\left[\int_{0}^{1}[\right. & -\frac{\sin (\pi x)}{\pi}\left(\tilde{u}^{2} / 2\right)_{x}+\pi \sin (\pi x)\left(\tilde{v}^{2} / 2\right)_{x} \\
& \left.\left.+2 \cos (\pi x) \tilde{u}^{2}\right] d x+\int_{0}^{1} \tilde{u}^{3}-\tilde{v}\left(\tilde{u}^{2} / 2\right)_{x}\right] d x .
\end{aligned}
$$

Integrating by parts, we find that

$$
\begin{aligned}
& \int_{0}^{1} \frac{\sin (\pi x)}{\pi}\left(\tilde{u}^{2} / 2\right)_{x}=-\int_{0}^{1} \frac{1}{2} \cos (\pi x) \tilde{u}^{2} d x \\
& \int_{0}^{1} \pi \sin (\pi x)\left(\tilde{v}^{2} / 2\right)_{x}=-\int_{0}^{1} \frac{\pi^{2}}{2} \cos (\pi x) \tilde{v}^{2} d x
\end{aligned}
$$

and

$$
\int_{0}^{1} \tilde{v}\left(\tilde{u}^{2} / 2\right)_{x}=-\int_{0}^{1} \frac{1}{2} \tilde{v}_{x} \tilde{u}^{2} d x=-\int_{0}^{1} \frac{1}{2} \tilde{u}^{3} d x .
$$

Hence we have that

$$
\frac{1}{2} \frac{d}{d t}\|\tilde{u}\|_{2}^{2}-\pi^{2}\|\tilde{u}\|_{2}^{2} \leq-\left\|\tilde{u}_{x}\right\|_{2}^{2}+A e^{-\pi^{2} t}\left[\frac{5}{2}\|\tilde{u}\|_{2}^{2}+\frac{\pi^{2}}{2}\|\tilde{v}\|_{2}^{2}+\int_{0}^{1} \frac{3}{2} \tilde{u}^{3} d x\right] .
$$

By the Poincaré inequality for functions with homogeneous Dirchlet boundary conditions and (5.2), we have

$$
\|\tilde{v}\|_{2}^{2} \leq \frac{1}{\pi^{2}}\|\tilde{u}\|_{2}^{2}
$$

and, also, by the Sobolev embedding theorem,

$$
\int_{0}^{1} \tilde{u}^{3} d x \leq \int_{0}^{1}|\tilde{u}|^{3} d x \leq\|\tilde{u}\|_{2}\|\tilde{u}\|_{4}^{2} \leq \frac{1}{C}\|\tilde{u}\|_{2}\left\|\tilde{u}_{x}\right\|_{2}^{2}
$$

Thus

$$
\frac{1}{2} \frac{d}{d t}\|\tilde{u}\|_{2}^{2} \leq \pi^{2}\|\tilde{u}\|_{2}^{2}-\left\|\tilde{u}_{x}\right\|_{2}^{2}+A e^{-\pi^{2} t}\left[3\|\tilde{u}\|_{2}^{2}+\frac{3}{2 C}\|\tilde{u}\|_{2}\left\|\tilde{u}_{x}\right\|_{2}^{2}\right]
$$

Hence

$$
\frac{1}{2} \frac{d}{d t}\|\tilde{u}\|_{2}^{2} \leq \pi^{2}\|\tilde{u}\|_{2}^{2}-\left[1-\frac{3 A e^{-\pi^{2} t}\|\tilde{u}\|_{2}}{2 C}\right]\left\|\tilde{u}_{x}\right\|_{2}^{2}+3 A e^{-\pi^{2} t}\|\tilde{u}\|_{2}^{2}
$$


so that, while

$$
e^{-\pi^{2} t}\|\tilde{u}\|_{2} \leq \frac{2 C \gamma}{3 A}
$$

it follows that

$$
\frac{1}{2} \frac{d}{d t}\|\tilde{u}\|_{2}^{2} \leq \gamma \pi^{2}\|\tilde{u}\|_{2}^{2}+3 A e^{-\pi^{2} t}\|\tilde{u}\|_{2}^{2}
$$

applying the Poincaré inequality for functions with homogeneous Neumann boundary conditions and zero mean. Integrating the inequality gives

$$
\|\tilde{u}\|_{2}^{2} \leq \exp \left[2 \gamma \pi^{2} t-\frac{6 A}{\pi^{2}}\left(e^{-\pi^{2} t}-1\right)\right]\|\tilde{u}(x, 0)\|_{2}^{2} .
$$

Thus

$$
\|\tilde{u}\|_{2}^{2} \leq e^{2 \gamma \pi^{2} t} e^{6 A / \pi^{2}}\|\tilde{u}(x, 0)\|_{2}^{2}
$$

and

$$
e^{-\pi^{2} t}\|\tilde{u}\|_{2} \leq e^{(\gamma-1) \pi^{2} t} e^{3 A / \pi^{2}}\|\tilde{u}(x, 0)\|_{2} \leq e^{3 A / \pi^{2}}\|\tilde{u}(x, 0)\|_{2} .
$$

Hence, choosing $\tilde{u}(x, 0)$ such that

$$
e^{3 A / \pi^{2}}\|\tilde{u}(x, 0)\|_{2} \leq \frac{2 C \gamma}{3 A},
$$

it is clear that (5.8) will hold for all $t \geq 0$, and it follows from (5.9) that

$$
\|\tilde{u}\|_{2}^{2} \leq 4 C^{2} \gamma^{2} e^{2 \gamma \pi^{2} t} / 9 A^{2}
$$

This completes the result.

Lemma 5.2. Assume that $\tilde{u}_{0} \in H^{2}(0,1)$. Given any $0<\gamma<1$, there exists $D$ independent of $A$, such that if

$$
\|\tilde{u}(x, 0)\|_{2} \leq e^{-7 A / \pi^{2}} 2 D \gamma / 3 A
$$

then (5.3)-(5.5) have a solution for all $t \geq 0$ and

$$
\left\|\tilde{u}_{x}(x, t)\right\|_{2} \leq e^{\gamma \pi^{2} t} 2 D \gamma / 3 A \quad \forall t \geq 0 .
$$

Proof. Taking the inner product of (5.3) with $-\tilde{u}_{x x}$ gives

$$
\begin{aligned}
\frac{1}{2} \frac{d}{d t}\left\|\tilde{u}_{x}\right\|_{2}^{2}-\pi^{2}\left\|\tilde{u}_{x}\right\|_{2}^{2} & \\
= & -\left\|\tilde{u}_{x x}\right\|_{2}^{2}+A e^{-\pi^{2} t}\left[\int_{0}^{1}\left[\frac{\sin (\pi x)}{\pi}\left(\tilde{u}_{x}^{2} / 2\right)_{x}-\pi \sin (\pi x) \tilde{v} \tilde{u}_{x x}-2 \cos (\pi x) \tilde{u} \tilde{u}_{x x}\right] d x\right. \\
& \left.+\int_{0}^{1}-\tilde{u}^{2} \tilde{u}_{x x}+\tilde{v}\left(\tilde{u}_{x}^{2} / 2\right)_{x}\right] d x .
\end{aligned}
$$


Now

$$
\begin{gathered}
\int_{0}^{1} \frac{\sin (\pi x)}{\pi}\left(\tilde{u}_{x}^{2} / 2\right)_{x} d x=-\int_{0}^{1} \frac{1}{2} \cos (\pi x) \tilde{u}_{x}^{2} d x \\
\int_{0}^{1} \pi \sin (\pi x) \tilde{v} \tilde{u}_{x x} d x=-\int_{0}^{1}\left[\pi \sin (\pi x) \tilde{u} \tilde{u}_{x}+\pi^{2} \cos (\pi x) \tilde{v} \tilde{u}_{x}\right] d x \\
\int_{0}^{1} \cos (\pi x) \tilde{u} \tilde{u}_{x x} d x=-\int_{0}^{1}\left[\cos (\pi x) \tilde{u}_{x}^{2}-\pi \sin (\pi x) \tilde{u} \tilde{u}_{x}\right] d x \\
\int_{0}^{1} \tilde{u}^{2} \tilde{u}_{x x} d x=-2 \int_{0}^{1} \tilde{u} \tilde{u}_{x}^{2} d x
\end{gathered}
$$

and

$$
\int_{0}^{1}\left(\tilde{u}_{x}^{2} / 2\right)_{x} d x=-\int_{0}^{1} \frac{\tilde{u} \tilde{u}_{x}^{2}}{2} d x .
$$

Hence, by Cauchy-Schwarz,

$$
\begin{array}{r}
\frac{1}{2} \frac{d}{d t}\left\|\tilde{u}_{x}\right\|_{2}^{2}-\pi^{2}\left\|\tilde{u}_{x}\right\|_{2}^{2} \leq-\left\|\tilde{u}_{x x}\right\|_{2}^{2}+A e^{-\pi^{2} t}\left[+\frac{1}{2}\left\|\tilde{u}_{x}\right\|_{2}^{2}+\pi\|\tilde{u}\|_{2}\left\|\tilde{u}_{x}\right\|_{2}\right. \\
\left.+\pi^{2}\|\tilde{v}\|_{2}\left\|\tilde{u}_{x}\right\|_{2}+2\left\|\tilde{u}_{x}\right\|_{2}^{2}+2 \pi\|\tilde{u}\|_{2}\left\|\tilde{u}_{x}\right\|_{2}+\frac{3}{2} \int_{0}^{1} \tilde{u} \tilde{u}_{x}^{2} d x\right] .
\end{array}
$$

Since $u_{0} \in H^{2}(0,1)$ we deduce from Theorem 3.1 that $u(\bullet, t) \in H^{2}(0,1)$ provided that a solution exists; thus $\left\|\tilde{u}_{x x}\right\|_{2}^{2}$ exists. Applying the Poincaré inequality to functions with Neumann and Dirichlet boundary conditions (using the integral constraint (5.6)), respectively, and the Sobolev embedding theorem, we obtain

$$
\begin{aligned}
\|\tilde{u}\|_{2} & \leq \frac{1}{\pi}\left\|\tilde{u}_{x}\right\|, \\
\|\tilde{v}\|_{2} & \leq \frac{1}{\pi}\|\tilde{u}\|,
\end{aligned}
$$

and

$$
\left|\int_{0}^{1} \tilde{u} \tilde{u}_{x}^{2} d x\right| \leq\|\tilde{u}\|_{2}\left\|\tilde{u}_{x}\right\|_{4}^{2} \leq \frac{1}{D}\|\tilde{u}\|_{2}\left\|\tilde{u}_{x x}\right\|_{2}^{2}
$$

Thus we have

$$
\begin{aligned}
& \frac{1}{2} \frac{d}{d t}\left\|\tilde{u}_{x}\right\|_{2}^{2}-\pi^{2}\left\|\tilde{u}_{x}\right\|_{2}^{2} \\
& \quad \leq-\left\|\tilde{u}_{x x}\right\|_{2}^{2}+A e^{-\pi^{2} t}\left[\frac{1}{2}\left\|\tilde{u}_{x}\right\|_{2}^{2}+2\left\|\tilde{u}_{x}\right\|_{2}^{2}+4\left\|\tilde{u}_{x}\right\|_{2}^{2}+\frac{3}{2 D}\|\tilde{u}\|_{2}\left\|\tilde{u}_{x x}\right\|_{2}^{2}\right] \\
& \quad \Rightarrow \frac{1}{2} \frac{d}{d t}\left\|\tilde{u}_{x}\right\|_{2}^{2} \leq \pi^{2}\left\|\tilde{u}_{x}\right\|_{2}^{2}-\left(1-\frac{3 A e^{-\pi^{2} t}}{2 D}\|\tilde{u}\|_{2}\right)\left\|\tilde{u}_{x x}\right\|_{2}^{2}+7 A e^{-\pi^{2} t}\left\|\tilde{u}_{x}\right\|_{2}^{2} .
\end{aligned}
$$

The same reasoning as in Lemma 5.3 yields the required result. The bounds on $\|u\|_{2}$ and $\left\|u_{x}\right\|_{2}$ imply an $H^{1}$ bound on the solution and the existence of a solution for all time $t \geq 0$ follows from Theorem 3.1.

We now obtain $L_{2}$ stability as required; in the following, $C$ and $D$ are given by the previous lemmas. 
Lemma 5.3. Let $u_{0} \in H^{2}(0,1)$. Given any $0<\gamma<\frac{1}{3}$ let

$$
\|\tilde{u}(x, 0)\|_{2} \leq \frac{2 \gamma e^{-4 A / \pi^{2}}}{3 A} \min \left\{e^{-3 A / \pi^{2}} D, C\right\} .
$$

Then (5.3)-(5.5) have a solution for all $t \geq 0$ satsifying

$$
\|\tilde{u}(x, t)\|_{2}^{2} \leq\|\tilde{u}(x, 0)\|_{2}^{2}+\frac{8 \gamma^{2}\left(3 A C^{2}+\gamma D^{2}\right)}{9 \pi^{2} A^{2}(1-3 \gamma)} \quad \forall t \geq 0 .
$$

Proof. Applying Lemmas 5.3 and 5.4 to (5.7) gives

$$
\frac{1}{2} \frac{d}{d t}\|\tilde{u}\|_{2}^{2} \leq \pi^{2}\|\tilde{u}\|_{2}^{2}-\left\|\tilde{u}_{x}\right\|_{2}^{2}+A e^{-\pi^{2} t}\left[3 e^{2 \gamma \pi^{2} t}(2 C \gamma / 3 A)^{2}+\frac{4 e^{3 \gamma \pi^{2} t} D^{2} \gamma^{3}}{9 A^{3}}\right] .
$$

Applying the Poincaré inequality for functions with Neumann boundary conditions and zero mean, we obtain

$$
\frac{1}{2} \frac{d}{d t}\|\tilde{u}\|_{2}^{2} \leq A e^{(3 \gamma-1) \pi^{2} t}\left[\frac{4 C^{2} \gamma^{2}}{3 A^{2}}+\frac{4 D^{2} \gamma^{3}}{9 A^{3}}\right]
$$

Integrating, we obtain

$$
\begin{aligned}
\|\tilde{u}\|_{2}^{2}-\|\tilde{u}(x, 0)\|_{2}^{2} & \leq\left[\frac{2 e^{\pi^{2}(3 \gamma-1) \pi^{2} t}}{(3 \gamma-1)}\left(\frac{4 C^{2} \gamma^{2}}{3 A^{2}}+\frac{4 D^{2} \gamma^{3}}{9 A^{3}}\right)\right]_{0}^{1} \\
& \leq \frac{2}{\pi^{2}(1-3 \gamma)}\left[\frac{4 C^{2} \gamma^{2}}{3 A}+\frac{4 D^{2} \gamma^{3}}{9 A^{2}}\right]=\frac{8 \gamma^{2}}{\pi^{2}(1-3 \gamma) 9 A^{2}}\left[3 A C^{2}+\gamma D^{2}\right] .
\end{aligned}
$$

This completes the proof.

We now show that, in fact, the solution is asymptotically stable.

LEMMA 5.4. Under the same conditions as Lemma 5.5,

$$
\int_{0}^{1} \tilde{u}(x, t) \cos (m \pi x) \rightarrow 0 \quad \text { as } t \rightarrow \infty
$$

uniformly in $m$, for all $m \geq 2$.

Proof. Let

$$
a_{m}=\int_{0}^{1} \tilde{u}(x, t) \cos (m \pi x) d x .
$$

Multiplying (5.3) by $\cos (m \pi x)$ and integrating by parts gives

$$
\begin{gathered}
\frac{d a_{m}}{d t}-\pi^{2} a_{m}=-m^{2} \pi^{2} a_{m}+A e^{-\pi^{2} t}\left[\int_{0}^{1} \frac{-1}{\pi} \sin (\pi x) \cos (m \pi x) \tilde{u}_{x}\right. \\
+\pi \sin (\pi x) \cos (m \pi x) \tilde{v}+2 \cos (\pi x) \cos (m \pi x) \tilde{u}] d x+\int_{0}^{1} \cos (m \pi x)\left[\tilde{u}^{2}-\tilde{v} \tilde{u}_{x}\right] d x .
\end{gathered}
$$

Using elementary trigonometric identities and integration by parts, it may be shown that

$$
\begin{gathered}
\int_{0}^{1} \frac{1}{\pi} \sin (\pi x) \cos (m \pi x) \tilde{u}_{x} d x=-\frac{(m+1)}{2} a_{m+1}+\frac{(m-1)}{2} a_{m-1} \\
\int_{0}^{1} \pi \sin (\pi x) \cos (m \pi x) \tilde{v} d x=\frac{a_{m+1}}{2(m+1)}-\frac{a_{m-1}}{2(m-1)}
\end{gathered}
$$


and

$$
\int_{0}^{1} 2 \cos (\pi x) \cos (m \pi x) \tilde{u} d x=a_{m+1}+a_{m-1}
$$

Thus we have

$$
\begin{aligned}
\frac{d a_{m}}{d t}= & \left(1-m^{2}\right) \pi^{2} a_{m}+A e^{-\pi^{2} t}\left[\frac{m+1}{2} a_{m+1}-\frac{m-1}{2} a_{m-1}+\frac{a_{m+1}}{2(m+1)}\right. \\
& \left.-\frac{a_{m-1}}{2(m-1)}+a_{m+1}+a_{m-1}+\int_{0}^{1} \cos (m \pi x)\left(\tilde{u}^{2}-\tilde{v} \tilde{u}_{x}\right) d x\right]
\end{aligned}
$$

Now,

$$
\begin{aligned}
\frac{1}{2} \frac{d}{d t}\left(a_{m}^{2}\right)= & \left(1-m^{2}\right) \pi^{2} a_{m}^{2} \\
& +A e^{-\pi^{2} t}\left[\left(1+\frac{m+1}{2}+\frac{1}{2(m+1)}\right) a_{m} a_{m+1}\right. \\
& \left.-\left(\frac{(m-1)}{2}+\frac{1}{2(m-1)}-1\right) a_{m} a_{m-1}\right]+a_{m} \int_{0}^{1} \cos (m \pi x)\left(\tilde{u}^{2}-\tilde{v} \tilde{u}_{x}\right) d x
\end{aligned}
$$

Completing the square and using the fact that $m \geq 2$ gives

$$
\begin{aligned}
\frac{1}{2} \frac{d}{d t}\left(a_{m}^{2}\right)= & \left(1-m^{2}\right) \pi^{2} a_{m}^{2}+A e^{-\pi^{2} t}\left[\left(\frac{m}{4}+1\right)\left(a_{m}^{2}+a_{m+1}^{2}\right)+\frac{3 m}{4}\left(a_{m}^{2}+a_{m-1}^{2}\right)\right] \\
& +A e^{-\pi^{2} t}\left|a_{m}\right|\left[\|\tilde{u}\|_{2}^{2}+\|\tilde{v}\|_{2}\left\|\tilde{u}_{x}\right\|_{2}\right]
\end{aligned}
$$

Now, by Lemma 5.5,

$$
a_{m}^{2} \leq \frac{1}{2}\|\tilde{u}\|_{2}^{2} \leq c_{1}(A)^{2} \quad \forall k \geq 0 .
$$

Also

$$
\|\tilde{v}\|_{2} \leq \frac{1}{\pi}\|\tilde{u}\|_{2} \leq \frac{\sqrt{(2)} c_{1}(A)}{\pi}
$$

and, by Lemma 5.4 ,

$$
\left\|\tilde{u}_{x}\right\|_{2} \leq \pi e^{\gamma \pi^{2} t} d_{1}(A) / \sqrt{(2)}
$$

for $0<\gamma<\frac{1}{3}$. Hence,

$$
\begin{aligned}
\frac{1}{2} \frac{d}{d t}\left(a_{m}^{2}\right)= & \left(1-m^{2}\right) \pi^{2} a_{m}^{2}+A e^{-\pi^{2} t}\left[2(m+1) c_{1}(A)^{2}\right. \\
& \left.+c_{1}(A)\left(2 c_{1}(A)^{2}+e^{\gamma \pi^{2} t} c_{1}(A) d_{1}(A)\right)\right]
\end{aligned}
$$

Thus

$$
\frac{1}{2} \frac{d}{d t}\left(a_{m}^{2}\right)=\left(1-m^{2}\right) \pi^{2} a_{m}^{2}+c_{2}(A) m e^{-(1-\gamma) \pi^{2} t}
$$

and integrating, we obtain

$$
\frac{d}{d t}\left[\exp \left(\left(m^{2}-1\right) \pi^{2} t\right) a_{m}^{2}(t)\right] \leq c_{2}(A) m e^{\left(m^{2}-2+\gamma\right) \pi^{2} t} .
$$


Thus we find that

$$
\begin{aligned}
a_{m}^{2}(t) & \leq e^{\left(1-m^{2}\right) \pi^{2} t} a_{m}^{2}(0)+\frac{m c_{2}(A) e^{-(1-\gamma) \pi^{2} t}}{m^{2}-2+\gamma} \\
& \leq e^{\left(1-m^{2}\right) \pi^{2} t} a_{m}^{2}(0)+c_{2}(A) e^{-(1-\gamma) \pi^{2} t}
\end{aligned}
$$

since $m \geq 2$. The result follows upon taking the limit $t \rightarrow \infty$.

Proof of Theorem III(b). Lemma 5.5 establishes the stability of the solution to perturbations in $L_{2}$ since $\gamma$ may be taken arbitrarily small. Lemma 5.6 proves asymptotic stability.

6. Behaviour for $\boldsymbol{\mu}>$ 1. Conjecture IV. We now briefly examine the case $\mu>1$. The results of the previous sections indicate that blow-up might not be expected to occur in this case. To verify this we have made extensive numerical calculations which demonstrate that when $\mu>1$, the function $u(x, t)$ decays to zero at a uniform exponential rate. To see this, in Figs. 6.1 and 6.2 we present the results of two calculations taking $\mu=1.25$ and $\mu=4$, respectively. In both cases the initial data was taken to be $100 \cos (\pi x)$ as in the previous calculations. In the figures we plot $\log (u(0, t))$ as a function of $t$ and it is clear that the decay is uniformly exponential in character and is more rapid initially when $\mu=4$.

In the special case of $\mu=4$ we may deduce that $u(x, t)$ decays to zero by using energy estimates similar to those derived in $\S 6$.

Proposition 6.1. If $\mu=4$ and $u(x, 0) \in H^{2}(0,1)$ then, for any $u_{0}$, the solution of (1.1)-(1.4) exists for all $t \geq 0$ and satisfies

$$
\|u(x, t)\|_{\infty} \rightarrow 0 \quad \text { as } \quad t \rightarrow \infty
$$

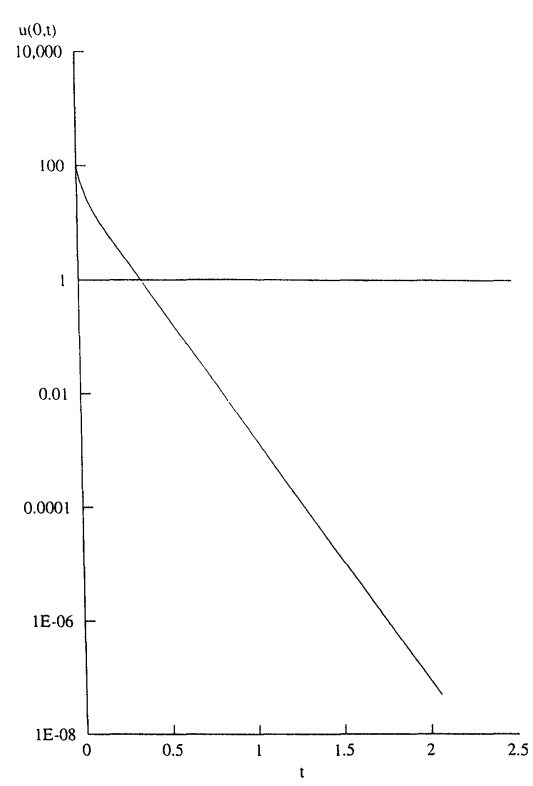

FIG. 6.1. The function $u(0, t)$ when $\mu=1.25$. 


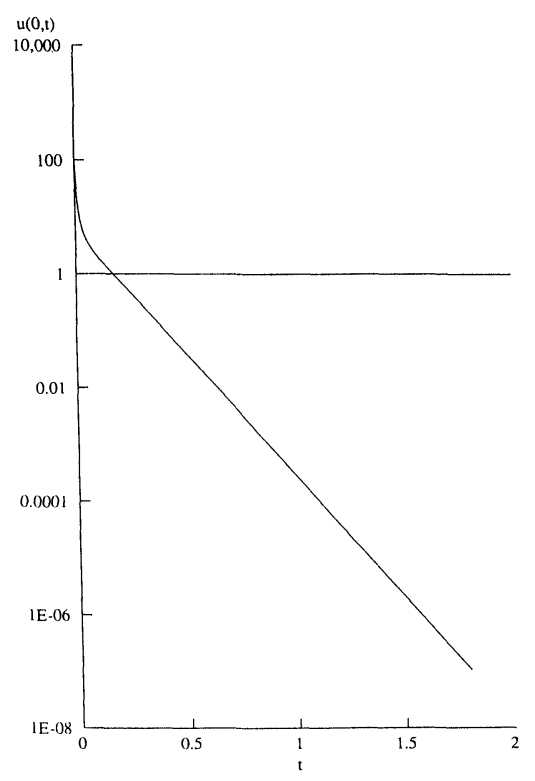

Fig. 6.2. The function $u(0, t)$ when $\mu=4$.

Proof. Multiplying (1.1) through by $u_{x x}$ and integrating, we obtain

$$
\frac{1}{2} \frac{d}{d t}\|u\|_{H^{1}}^{2}=-\left\|u_{x x}\right\|_{2}^{2}-\left(2-\frac{\mu}{2}\right) \int_{0}^{1} u u_{x}^{2} d x .
$$

Since $u(x, 0) \in H^{2}(0,1)$, it remains in this space for all time by Theorem 3.1. The proof follows directly from (6.1) since by setting $\mu=4$ and applying the Poincaré inequality, we obtain

$$
\frac{1}{2} \frac{d}{d t}\|u\|_{H^{1}}^{2} \leq-\pi^{2}\|u\|_{H^{1}}^{2}
$$

Noting that the spatial dimension of the problem is one, the result follows by Sobolev embedding.

Acknowledgments. We are grateful to J. T. Stuart for related discussions and for suggesting the similarity solution outlined in $\S 2$, and to V. Galaktionov, E. Suli, and M. Floater for useful advice.

\section{REFERENCES}

[ABG] N. D. Alikakos, P. W. Bates, AND C. P. GRANT, Blow-up for a diffusion-advection equation, Proc. Roy. Soc. Edinburgh, 113A (1989), pp. 181-189.

[BDS1] C. J. Budd, J. W. Dold, AND A. M. StuART, Blowup in a system of partial differential equations with conserved first integral, SIAM J. Appl. Math., 53 (1993), pp. 718-742.

[BDS2] — Blowup in a System of Partial Differential Equations with Convection, Bristol University Report AM-93-06, U.K., 1993.

[Ch] S. Childress, Chemotactic collapse in two dimensions., Lect. Notes Biomath, 55 (1984), pp. 6166.

[CISY] S. Childress, G. R. Ierley, E. A. Spiegel, And W. R. Young, Blow-up of unsteady twodimensional Euler and Navier-Stokes solutions having stagnation point form, J. Fluid Mech., 203 (1989), pp. 1-23. 
[Cox] S. Cox, Two dimensional flow of a viscous fluid in a channel with porous walls, J. Fluid Mech., 227 (1991), pp. 1-33.

[DOK] E. Doedel And J. P. Kervenes, AUTO: Software for Continuation and Bifurcation in ODE's, Tech. Report, California Institute of Technology, Pasadena, 1986.

[Hen] D. HenRY, Geometric Theory of Semilinear Parabolic PDEs, Lecture Notes Math. 840, Springer-Verlag, New York, Berlin, 1980.

[Kat] T. KATO, On the classical solution of the two-dimensional, non-stationary Euler equation, Arch. Rat. Mech. Anal., 25 (1967), pp. 188-200.

[LAF] A. A. LACEY, AND A. FRIEDMAN, Blow-up of solutions of semilinear parabolic equations, J. Math. Anal. Appl., 132 (1988), pp. 171-186.

[Paz] A. PAZY, Semigroups of linear operators and applications to partial differential Equations, Appl. Math. Sci. 44, Springer-Verlag, New York, Berlin, 1983.

[RS] J. Rubenstein AND P. STERnBerg, Non-local reaction-diffusion equations and nucleation, IMA J. Appl. Math., 48 (1991), pp. 249-264.

[Stu] J. T. STUART, Nonlinear Euler partial differential equations: singularities in their solution, Proc. Symp. Honoring C. C. Lin, D. J. Benney, F. H. Shu, and Yuan Chi, eds., (Singapore), World Scientific Press, 1988, pp. 81-95.

[Tay] G. I. TAYLOR, On the decay of vortices in a viscous fluid, Philosophical Magazine, 46 (1923), pp. 671-674.

[Tem] R. TemAM, Navier-Stokes Equations and Nonlinear Functional Analysis, Society for Industrial and Applied Mathematics, Philadelphia, PA, 1983.

[ZDB] M. B. Zaturska, P. G. Drazin, And W. H. H. Banks, On the flow of a viscous fluid driven along a channel by suction at porous walls, Fluid Dynamic Res., 4 (1988), pp. 151-178. 